Short Communication

\title{
The Hardy-Weinberg principle
}

\author{
Alan E. Stark \\ University of New South Wales, School of Community Medicine, Kensington, Australia.
}

\begin{abstract}
Hardy-Weinberg genotypic proportions can be maintained in a population under non-random mating. A compact formula gives the proportions of mating pair types. These are illustrated by some simple examples.
\end{abstract}

Key words: Hardy-Weinberg equilibrium, non-random mating.

Received: March 11, 2005; Accepted: May 5, 2005.

Consider a population with respect to a single locus having alleles $a$ and $A$ with respective frequencies $q$ and $p$. Denote frequencies of genotypes $a a, a A$ and $A A$ by $f_{0}, f_{l}$ and $f_{2}$. Denote the $3 \times 3$ matrix of mating-pair frequencies by $\left[f_{i j}\right]$, $i=0,1,2 ; j=0,1,2$. Suppose the population has attained Hardy-Weinberg $(\mathrm{H}-\mathrm{W})$ proportions $f_{0}=q^{2}, f_{1}=2 p q, f_{2}=p^{2}$.

Then $\mathrm{H}-\mathrm{W}$ proportions are maintained if the elements of $\left[f_{i j}\right]$ are given by

$$
f_{i j}=f_{i} f_{j}\left(1+v e_{i} e_{j}\right),
$$

where $e_{0}=-p / q, e_{1}=1$ and $e_{2}=-q / p$.

Random mating is defined by matrix (1) with $v=0$, i.e. $f_{i j}=f_{i} f_{j}$.

Under the stated conditions, the sum of elements in the first (zero) row of $\left[f_{i j}\right]$ is $f_{0}=q^{2}$. This sum is also the frequency of type $a a$ in the offspring, as can be seen by summing the appropriately weighted terms of $\left[f_{i j}\right]$, noting that $f_{11}=4 f_{02}$

The applicable interval of $v$, as a function of $q$, is governed by the need for the $f_{i j}$ to be non-negative. Without loss of generality, consider $q$ in the range $0<q \leq 1 / 2$. Then the interval containing permissible values of $v$ is $\left(-q^{2} / p^{2}, q / p\right)$. At the lower limit, reading from left to right and from top to bottom row, the elements of $\left[f_{i j}\right]$ are $0,2 q^{3}, q^{2}(p-q), 2 q^{3}$, $4 q^{2}(p-q), 2 q\left(p^{3}+q^{3}\right), q^{2}(p-q), 2 q\left(p^{3}+q^{3}\right),(p-q)\left(p^{2}+q^{2}\right)$. For the upper limit, the values are $q^{3}, 0, p q^{2}, 0,4 p q^{2}, 2 p q(p-$ $q), p q^{2}, 2 p q(p-q), p\left(p^{3}+q^{3}\right)$. Thus it can be seen that the mating scheme defined by (1) gives a wide spectrum of non-random mating which maintains $\mathrm{H}-\mathrm{W}$ proportions, i.e. the "Hardy-Weinberg Principle" is more general than is usually envisaged.

Stark (1980) gives a more general mating scheme which subsumes (1). Li (1988) showed that random mating is a sufficient condition, not a necessary one, for the attainment of the Hardy Weinberg proportions, but here we provide for the first time a truly generalized mathematical argumentation to prove the fact. Stark (1977 a,b) give a more detailed description of the underlying mating model and several diagrams which illustrate the ranges of applicability of formula (1).

\section{References}

Li CC (1988) Pseudo-random mating populations. In celebration of the $80^{\text {th }}$ anniversary of the Hardy-Weinberg law. Genetics 119:731-737.

Stark AE (1977a) Models of correlation between mates and relatives and some applications. Unpublished Ph.D. Thesis, School of Community Medicine, The University of New South Wales, Kensington, Australia.

Stark AE (1977b) Dwa uogólnienia prawa Hardy'ego-Weinberga w genetyce populacji (The Hardy-Weinberg Law of Population Genetics and two Generalizations). Matematyka Stosowana IX:123-137.

Stark AE (1980) Inbreeding systems: Classification by a canonical form. J Math Biol 10:305.

Editor: Fábio de Melo Sene 